\title{
Identifying pulsation modes from two-passband photometry
}

\author{
J. Daszyńska-Daszkiewicz
}

Instytut Astronomiczny, Uniwersytet Wrocławski, ul. Kopernika 11, Poland

\section{Abstract}

I discuss a prospect for mode identification from two-passband photometry of forthcoming BRITE space mission. Examples of photometric diagnostic diagrams are shown for three types of main sequence pulsating variables: $\beta$ Cephei, Slowly Pulsating B-type and $\delta$ Scuti stars. I consider also taking into account the radial velocity data from simultaneous spectroscopy, which can be carried out from the ground. With such observations, much better discrimination of the spherical harmonic degree, $\ell$, can be accomplished and more constraints on stellar parameters and input physics can be derived.

\section{Introduction}

Nowadays space based observations allow detecting oscillation modes with lower and lower photometric amplitudes. We are already at the detecton threshold of the order of $10^{-5}-10^{-6}$ mag, resulting in a growing number of frequency peaks. There are many examples of excellent work both observational and theoretical based on WIRE and MOST data (e.g. Bruntt et al. 2007, Walker et al. 2005, Barban et al, 2007, Saio et al. 2007). Now we expect similar results from the just initiated COROT mission.

However, for using these rich frequency data for asteroseismic modelling, mode identification is a prerequisite. In the case of main sequence pulsators, we are still far from obtaining very regular patterns in the oscillation spectra, which could help in solving the problem. We are also far from nonlinear theory, which would answer a question about mode selection mechanism. The main unresolved problem is why most of the theoretically unstable modes are not observed. Nowakowski (2005) suggested that the dominant effect of limiting the mode amplitude is a collective saturation of the opacity driving mechanism, instead of a resonant mode coupling.

In order to identify modes in $\beta$ Cephei, $\delta$ Scuti or Slowly Pulsating Btype stars, we need additional observables from multicolour photometry or/and 
spectroscopy. The photometric method of mode identification consists in using amplitude ratios and phase differences in different passbands; it is based on the semi-analytical formula for the light variation due to linear pulsation derived by Dziembowski (1977). Balona \& Stobie (1979) showed that modes with different values of $\ell$ are located in different regions on the amplitude ratios $v s$ phase differences diagram. Since then, the method has been applied to various types of pulsating variables by many authors (Watson 1988, Garrido et al. 1990, Heynderickx et al. 1994). The next important improvement was including nonadiabatic calculations by Cugier, Dziembowski \& Pamyatnykh (1994), who applied the method to $\beta$ Cep stars. Then, Balona \& Evers (1999) emphasized the problem of very high sensitivity of photometric amplitudes and phases to the treatment of convection in the case of $\delta$ Sct stars. A photometric identification of $\ell$ for SPB stars, based on nonadiabatic calculations, was performed by Townsend (2002). All these works were done by assuming that the rotation does not influence pulsation. However, main sequence pulsators are very often rapid rotators. The next step in developing the method was its extension to close frequency modes coupled by a fast rotation by Daszyńska-Daszkiewicz et al. (2002). The photometric method in the version for long-period g-modes in rotating stars, for which perturbation approach is no longer adequate, was formulated by Townsend (2003a) and Daszyńska-Daszkiewicz et al. (2007).

A few years ago, Daszyńska-Daszkiewicz et al. (2003,2005a) proposed a new method of the identification of $\ell$, which uses the amplitudes and phases themselves and combines photometry, and radial velocity data. The method allows also to extract simultaneously a new asteroseismic probe, which yields constraints on stellar parameters and input physics, e.g., convection, opacities.

BRITE (BRIght Target Explorer) is the first space-based mission which will perform two-colour photometry of bright stars. It will give an opportunity of not only detecting low-level oscillations but also of identifying their degrees $\ell$. However, much more could be achieved, if ground-based spectroscopic observations are organized simultaneously. Then another type of information, contained in the radial velocity and line profile variations, would be supplied.

The aim of this paper is to show what can be done for mode identification from observations with the two BRITE passbands. I will recall the basic formulae and show examples of photometric diagnostic diagrams for models of $\beta$ Cephei, SPB and $\delta$ Scuti stars. Then I will present diagrams which include amplitudes and phases from photometric and radial velocity variations. Finally, I will discuss uncertainties arising from effects of rotation, convection and atmospheric models. A summary and conclusions are given in the last section. 


\section{Mode identification from photometry}

In order to calculate photometric amplitudes and phases, two inputs are needed. They come from

- nonadiabtic theory of stellar pulsation,

- models of stellar atmospheres.

We assume linear pulsation theory, which is adequate because of small mode amplitudes in main sequence pulsators, and we use temporally static, plane parallel atmosphere, which is justified because the eigenfunctions of the considered modes are nearly constant in the atmosphere.

Let us consider a pulsation mode in the zero-rotation approximation, the geometry of which can be described by a single spherical harmonic, $Y_{\ell}^{m}$, with the degree $\ell$ and the azimuthal order $m$. The shape of the radial eigenfunctions of the mode are determined by its radial order $n$. Then the local radial displacement is given by

$$
\frac{\delta r}{R}=\varepsilon Y_{\ell}^{m}(\theta, \phi) \mathrm{e}^{-\mathrm{i} \omega t},
$$

where $\varepsilon$ is the intrinsic mode amplitude, $\omega$ is the angular pulsation frequency, which of course depends on $(n, \ell, m)$; other symbols have their usual meanings. The corresponding changes of the bolometric flux, $\mathcal{F}_{\text {bol }}$, and the local gravity, $g$, are given by

$$
\frac{\delta \mathcal{F}_{\text {bol }}}{\mathcal{F}_{\text {bol }}}=\varepsilon f Y_{\ell}^{m}(\theta, \phi) \mathrm{e}^{-\mathrm{i} \omega t},
$$

and

$$
\frac{\delta g_{\mathrm{eff}}}{g_{\mathrm{eff}}}=-\varepsilon\left(2+\frac{\omega^{2} R^{3}}{G M}\right) Y_{\ell}^{m}(\theta, \phi) \mathrm{e}^{-\mathrm{i} \omega t} .
$$

The complex parameter, $f$, describes the ratio of the radiative flux perturbation to the radial displacement at the level of the photosphere and it is obtained from nonadiabatic calculations.

The complex photometric amplitudes of the light variation in the passband $\lambda$ due to a pulsation mode with frequency $\omega$ can be written in the following form

$$
\mathcal{A}_{\lambda}(i)=-1.086 \varepsilon Y_{\ell}^{m}(i, 0) b_{\ell}^{\lambda}\left(D_{1, \ell}^{\lambda}+D_{2, \ell}+D_{3, \ell}^{\lambda}\right)
$$

where

$$
\begin{gathered}
D_{1, \ell}^{\lambda}=\frac{1}{4} f \frac{\partial \log \left(\mathcal{F}_{\lambda}\left|b_{\ell}^{\lambda}\right|\right)}{\partial \log T_{\mathrm{eff}}}, \\
D_{2, \ell}=(2+\ell)(1-\ell),
\end{gathered}
$$




$$
D_{3, \ell}^{\lambda}=-\left(2+\frac{\omega^{2} R^{3}}{G M}\right) \frac{\partial \log \left(\mathcal{F}_{\lambda}\left|b_{\ell}^{\lambda}\right|\right)}{\partial \log g_{\text {eff }}^{0}}
$$

and $i$ is the inclination angle. The partial derivatives of $\log \left(\mathcal{F}_{\lambda}\left|b_{\ell}^{\lambda}\right|\right)$ over effective temperature and gravity are derived from atmospheric models and

$$
b_{\ell}^{\lambda}=\int_{0}^{1} h_{\lambda}(\mu) \mu P_{\ell}(\mu) d \mu,
$$

is the disc-averaging factor, containing the information about the visibility of the mode with a given degree $\ell$. The integrals $b_{\ell}^{\lambda}$ are weighted by the limbdarkening law, $h_{\lambda}(\mu)$. The term $D_{1, \ell}^{\lambda}$ describes the temperature effects, the term $D_{2, \ell}$ stands for the geometrical effects, and the influence of gravity changes is contained in the term $D_{3, \ell}^{\lambda}$. The terms $D_{1, \ell}^{\lambda}$ and $D_{3, \ell}^{\lambda}$ include the perturbation of the limb-darkening, and their $\ell$-dependence arises from the nonlinearity of the limb-darkening law.

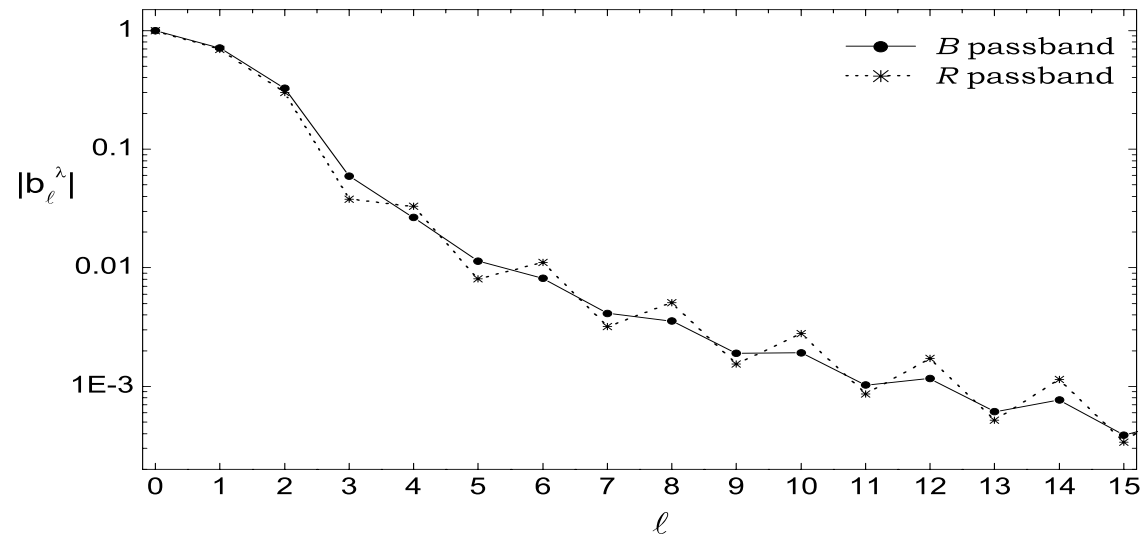

Figure 1: The disc-averaging factor, $b_{\ell}^{\lambda}$, as a function of the spherical harmonic degree, $\ell$, for the $B R$ Johnson filters. The adopted stellar parameters are $\log T_{\text {eff }}=3.875$ and $\log g=4.0$.

The amplitudes and phases of the light variation are given by $\left|\mathcal{A}_{\lambda}\right|$ and $\arg \left(\mathcal{A}_{\lambda}\right)$, respectively. Having these numbers, we can construct photometric diagnostic diagrams in the form $A_{x} / A_{y}$ vs. $\varphi_{x}-\varphi_{y}$, where $x$ and $y$ denote passbands. These observables are independent of the intrinsic amplitude, $\varepsilon$, inclination angle, $i$, and azimuthal order, $m$, because the product $\varepsilon Y_{\ell}^{m}$ drops out. These is an advantage for the identification of $\ell$ but also a disadvantage because the order $m$ is beyond of the reach of the photometric method. 
In this section I will give examples of photometric diagrams in the $B R$ Johnson filters which are not very different from the BRITE passbands: BT1 (390-460 nm) and BT2 (550-700 nm). The well known property of the factor $b_{\ell}^{\lambda}$ is that it decreases very rapidly with growing degree $\ell$; this can be seen from Fig. 1. In this paper I will consider modes with degrees up to $\ell=6$. All calculations were done with the Warsaw-New Jersey evolutionary code and nonadiabatic pulsation code of Dziembowski (1977). I used OPAL opacity tables of Iglesias \& Rogers (1996) and the solar chemical composition of Grevesse \& Noels (1993), assuming the metallicity $Z=0.02$. I adopted Kurucz atmospheric models in the NOVER-ODFNEW version (Castelli \& Kurucz 2004), which have more smooth flux derivatives than the standard Kurucz models, and the Claret nonlinear formula for the limb-darkening law. The standard atmospheric metallicity, $[\mathrm{m} / \mathrm{H}]=0.0$, and the microturbulence velocity, $\xi_{t}=2$ $\mathrm{km} / \mathrm{s}$, were assumed.

\section{$\beta$ Cephei pulsators}
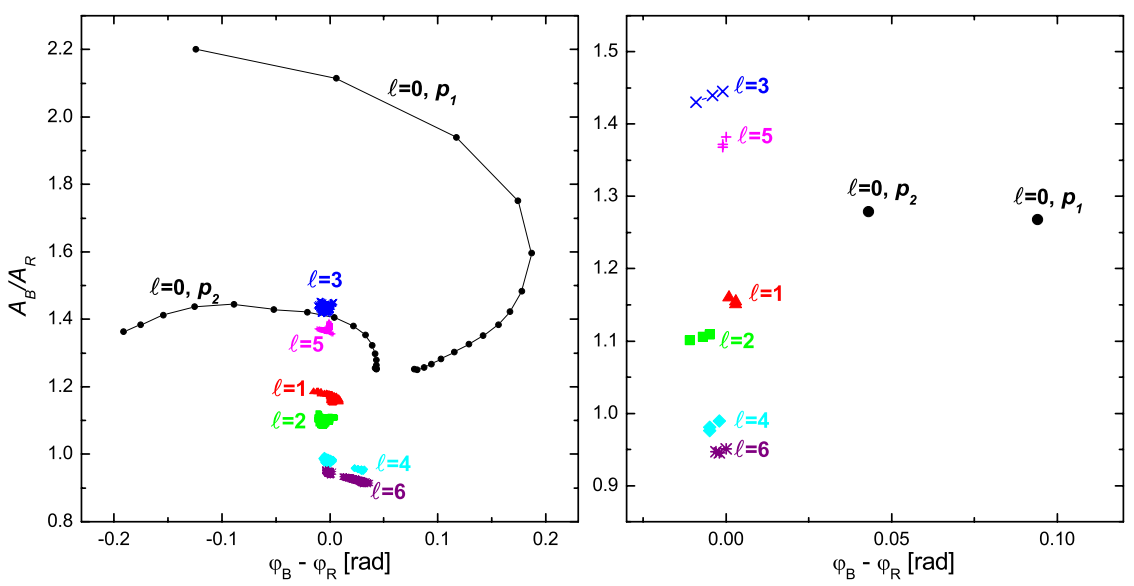

Figure 2: The locations of unstable modes with degrees $\ell$ up to 6 for $\beta$ Cephei star models of $12 M_{\odot}$ on the diagnostic diagrams involving Johnson $B$ and $R$ filters. The left panel contains all models in the main sequence evolutionary phase, and the right panel, the model with $\log T_{\text {eff }}=4.400$ and $\log g=3.89$.

I considered stellar models with a mass of $M=12 M_{\odot}$ during main sequence phase of evolution, corresponding to the temperature range of $\log T_{\text {eff }}=4.445-$ 4.347. In Fig. 2, the photometric diagram in the $B R$ passband is presented. The left panel shows all modes which become unstable between ZAMS and 
TAMS and the right panel shows unstable modes for only one stellar model with $\log T_{\text {eff }}=4.400$ and $\log g=3.89$. Modes with different degrees $\ell$ are located in separated regions. The radial modes are spread over a wide range of the amplitude ratios and phase differences, whereas the nonradial modes are concentrated in small areas. This behaviour results from different contributions of the temperature $\left(D_{1, \ell}^{\lambda} f\right)$ and the geometrical effects $\left(D_{2, \ell}\right)$ to the light variation (Daszyńska-Daszkiewicz et al. 2002)

\section{Slowly Pulsating B-type stars}

In order to calculate SPB oscillation, I chose the models with $M=5 M_{\odot}$ on

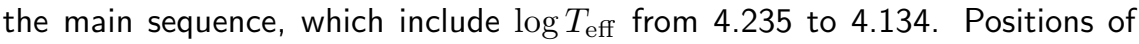
modes with different values of $\ell$ on the photometric $B R$ diagram are shown in Fig. 3. Again, the left panel contains all unstable modes, and the right panel, one model with $\log T_{\text {eff }}=4.195$ and $\log g=4.02$. The well-known property of this type of diagrams is the zero phase difference and the same amplitude ratio for all modes with $\ell=1$. This is because the light variation of the $\ell=1$ mode in SPB models is totaly dominated by the temperature effects. We can see an overlapping of domains with different values of $\ell$. This can be partly removed by considering only one model (the right panel). Another instructive
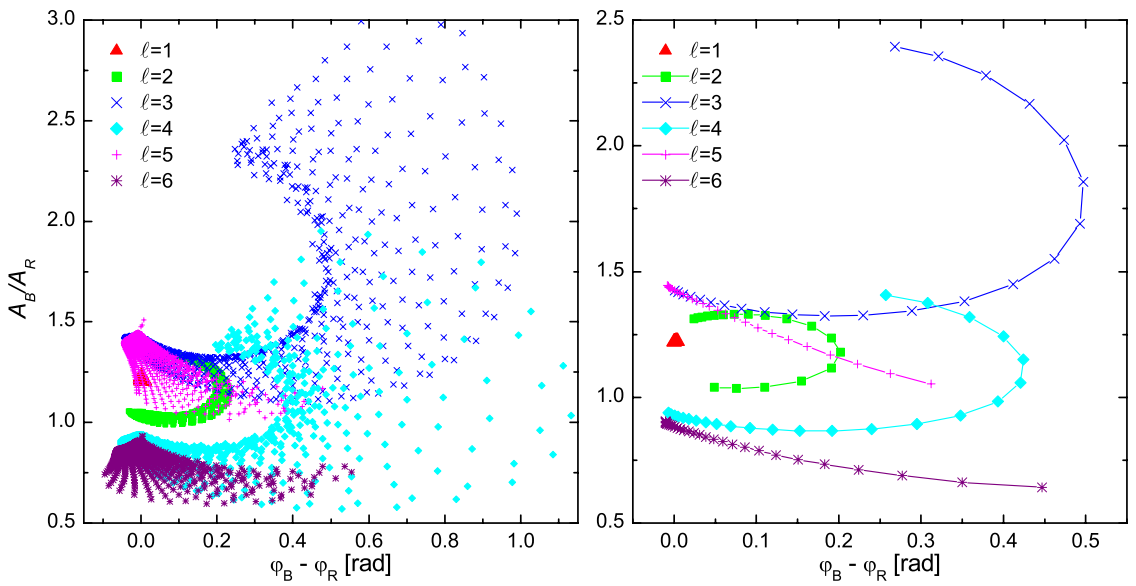

Figure 3: The same as in Fig. 2 but for Slowly Pulsating B-type star models of 5 $M_{\odot}$. The left panel contains all models in the main sequence evolutionary phase and the right panel, the model with $\log T_{\text {eff }}=4.195$ and $\log g=4.02$.

information can be drawn from instability conditions. Fig. 4 shows the amplitude ratio and the phase difference as a function of frequency for the model with 

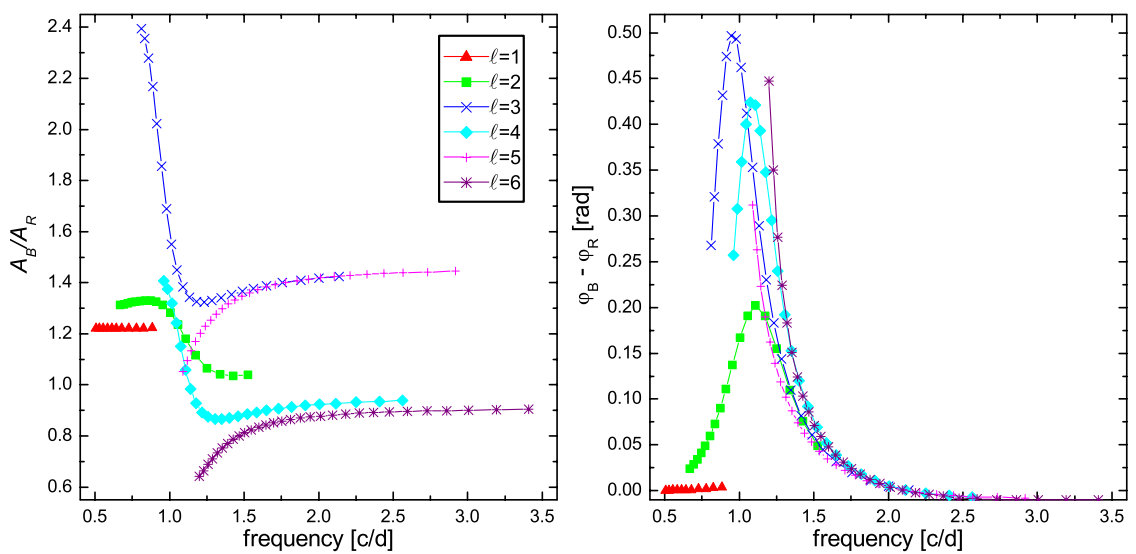

Figure 4: Amplitude ratios (on the left) and phase differences (on the right) in the $B R$ bands as a function of oscillation frequency for the SPB model with $\log T_{\text {eff }}=4.195$ and $\log g=4.02$.

$\log T_{\text {eff }}=4.195$ and $\log g=4.02$. As one can see, modes with $\ell=1$ are unstable only for the lowest frequencies $(\nu<0.9 \mathrm{c} / \mathrm{d})$. The instability is shifted to the higher frequencies for higher $\ell$-modes, e.g., the $\ell=5$ modes become unstable for $\nu>1.1 \mathrm{c} / \mathrm{d}$.

\section{$\delta$ Scuti pulsators}

As representatives of $\delta$ Sct pulsator, I took models with $M=2 M_{\odot}$ in the main sequence phase. The corresponding $\log T_{\text {eff }}$ range is $(3.963,3.854)$. All calculations were made under assumptions of the mixing length theory and the convective flux freezing approximation. The mixing length parameter was $\alpha_{\text {conv }}=0.0$, i.e., an assumption of inefficient convective transport. The photometric $B R$ diagram for this type of pulsators is presented in Fig. 5. All unstable modes of $M=2 M_{\odot}$ models are shown on the left hand side, and modes in the $\left(\log T_{\text {eff }}=3.909, \log g=4.02\right)$ model on the right hand side. As we can see, there is some overlap, especially for the $\ell \leq 2$ modes. Fixing the model parameters helps in removing this ambiguity.

The common opinion about $\delta$ Sct pulsation modes is that the main information about the $\ell$ values is contained in the phase differences. In fact, this is true only for low degree modes with $\ell \leq 2$, but for higher $\ell$ 's we can learn much also from the amplitude ratios. In Fig. 6, the amplitude ratio (the left panel) and phase difference (the right panel) as a function of oscillation frequency are depicted. As we can see, not much can be we achieved for low degree modes 

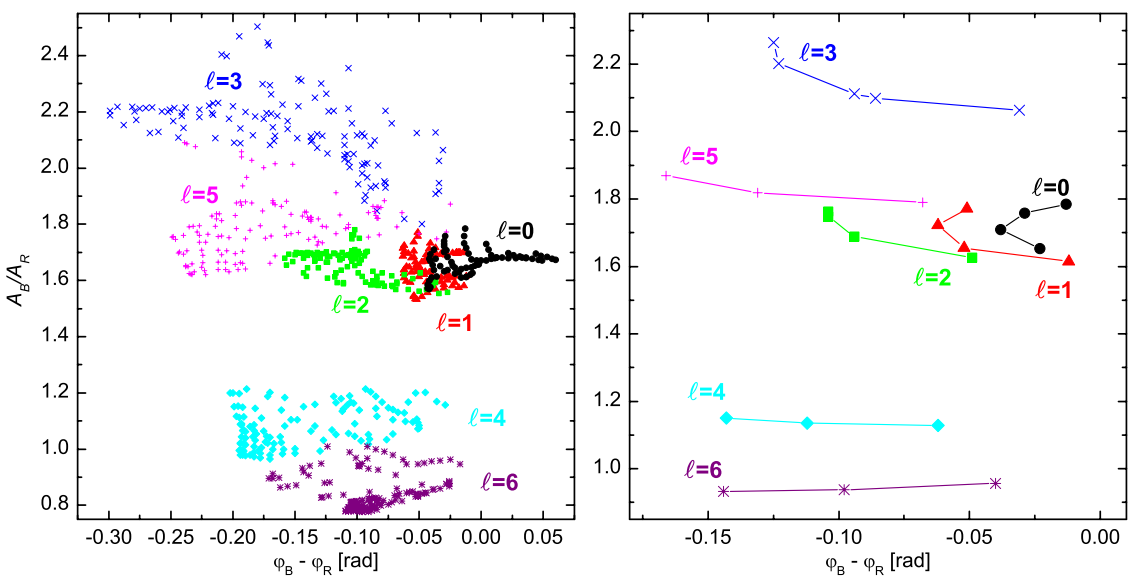

Figure 5: The same as in Fig. 2 but for $\delta$ Scuti star models of $2 M_{\odot}$. The left panel containes all models in the main sequence evolutionary phase, and the right panel, the model with $\log T_{\text {eff }}=3.909$ and $\log g=4.02$.
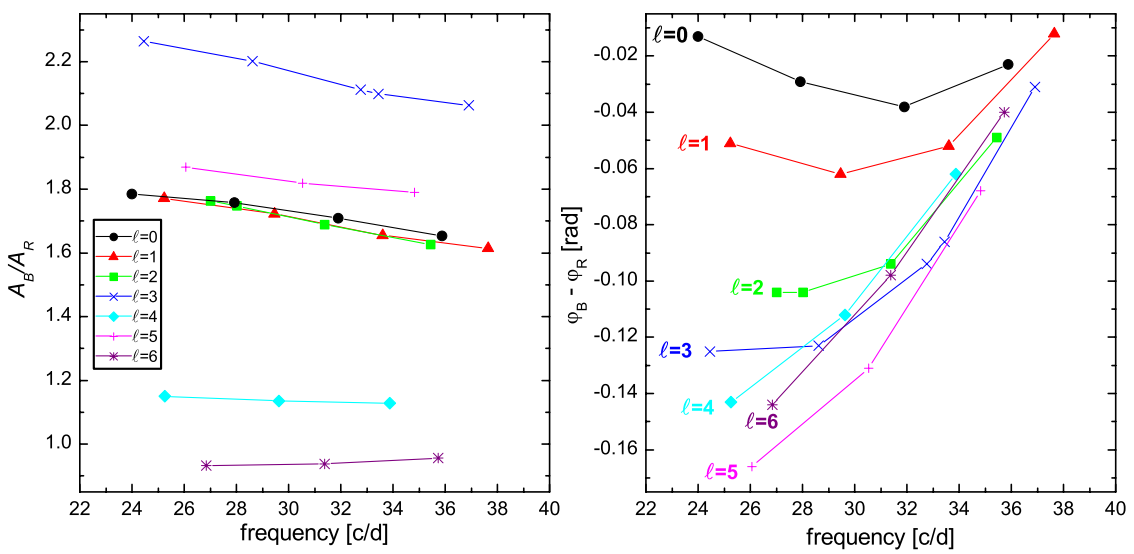

Figure 6: Amplitude ratios (on the left) and phase differences (on the right) in the $B R$ bands as a function of oscillation frequency for the $\delta$ Sct model with $\log T_{\text {eff }}=3.909$ and $\log g=4.02$.

also from the $A_{B} / A_{B} v s$. frequency plot because the $\ell \leq 2$ modes are excited with very close frequencies. Another interesting property to note is that for very high frequency mode $(\nu>35 \mathrm{c} / \mathrm{d})$, the phase differences are almost the same for all degrees $\ell$. 


\section{Adding radial velocity measurements}

The radial velocity variation averaged over stellar disc is expressed by the wellknown Dziembowski's (1977) formula

$$
V_{\text {rad }}(i)=\mathrm{i} \omega R \varepsilon Y_{\ell}^{m}(i, 0)\left(u_{\ell}^{\lambda}+\frac{G M}{R^{3} \omega^{2}} v_{\ell}^{\lambda}\right)
$$

where

$$
u_{\ell}^{\lambda}=\int_{0}^{1} h_{\lambda}(\mu) \mu^{2} P_{\ell}(\mu) d \mu
$$

and

$$
v_{\ell}^{\lambda}=\ell \int_{0}^{1} h_{\lambda}(\mu) \mu\left(P_{\ell-1}(\mu)-\mu P_{\ell}(\mu)\right) d \mu .
$$

From observations, the radial velocity variations are determined by calculating the first moment, $\mathcal{M}_{1}^{\lambda}$, of a well isolated spectral line.

In this section, I show examples of diagnostic diagrams constructed from the radial velocity variation and the light variation in the Johnson $R$ filter. Unstable oscillation modes for $\beta$ Cep, SPB and $\delta$ Sct star models considered in the previous section are presented in Fig 7,8 and 9 , respectively. As we can
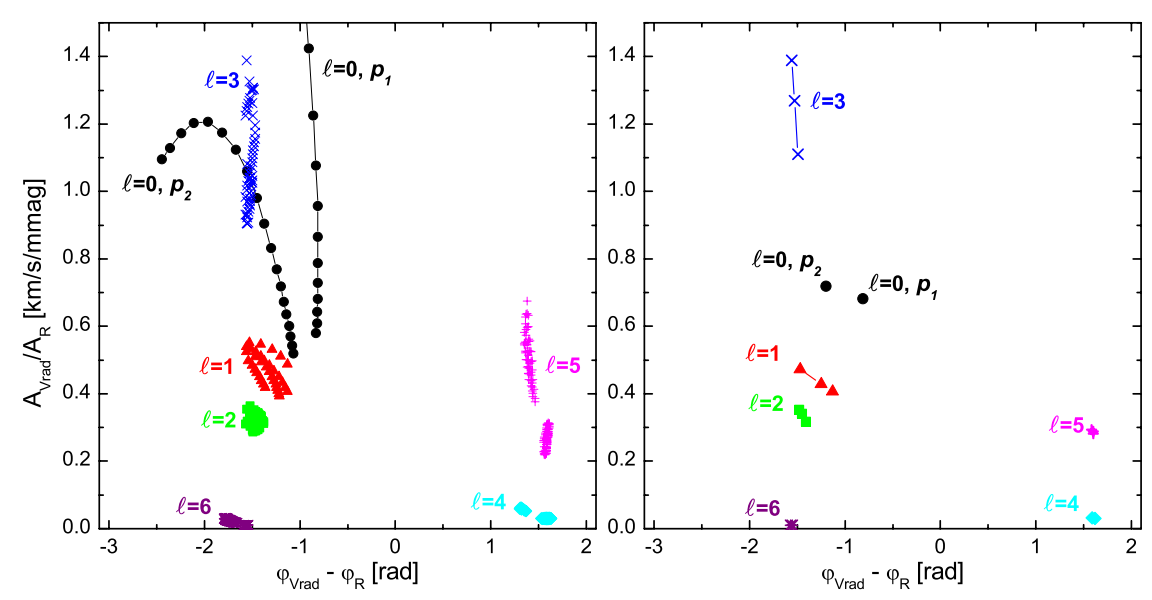

Figure 7: The location of unstable modes with degrees $\ell$ up to 6 for $\beta$ Cep models of $12 M_{\odot}$ on the diagnostic diagrams constructed with amplitudes and phases for the $R$ passband and the radial velocity variations. As in Fig. 2, the left panel shows all main sequence models, and the right panel, one model with $\log T_{\text {eff }}=4.400$ and $\log g=3.89$. 

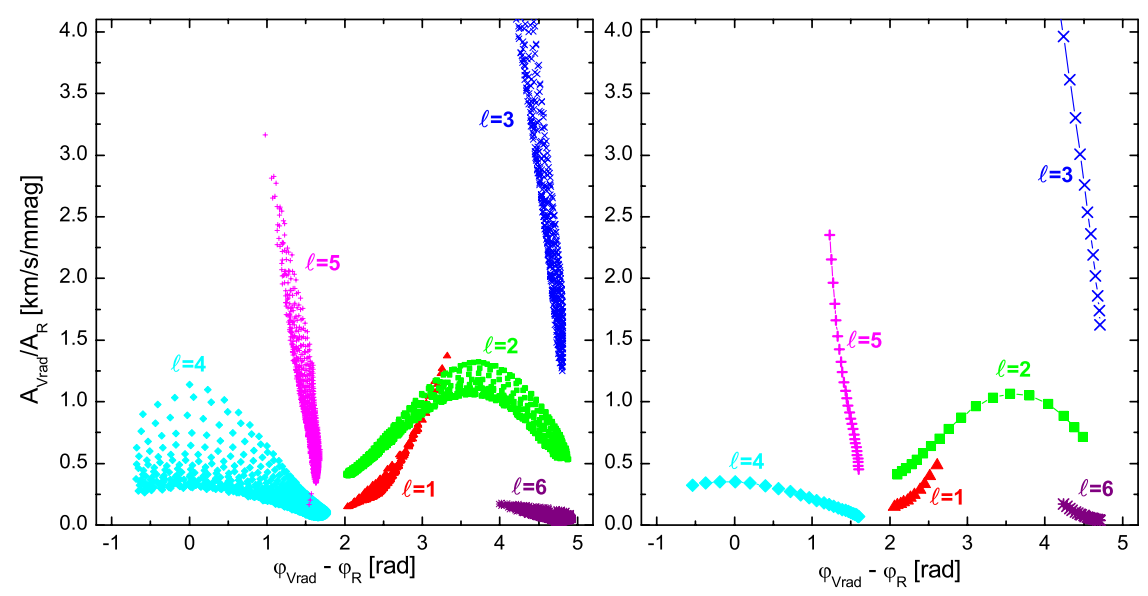

Figure 8: The same as in Fig. 7 but for SPB models of $5 M_{\odot}$. In the right panel unstable modes for the model with $\log T_{\text {eff }}=4.195$ and $\log g=4.02$ are plotted.
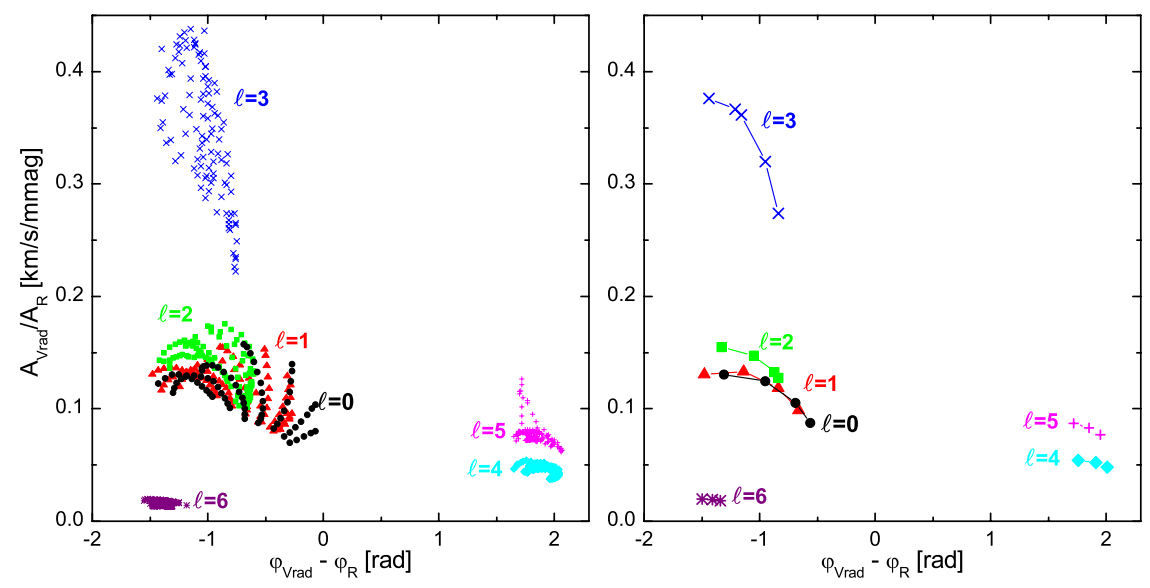

Figure 9: The same as in Fig. 7 but for $\delta$ Sct models of $2 M_{\odot}$. In the right panel unstable modes for the model with $\log T_{\text {eff }}=3.909$ and $\log g=4.02$ are plotted.

see, the configuration of $\ell$ modes domains differs from that in the photometric diagrams. In particular, there are larger phase differences between light and radial velocity variation than between two passnands.

Moreover, with two photometric passbands and the radial velocity data, one can apply the method of simultaneous extracting from observations the 
degree $\ell$ and the nonadiabatic parameter $f$. Determination of the empirical value of $f$ allows to avoid, in the process of the $\ell$-identification, the input from pulsation models, which still needs many improvements, for example the pulsation-convection interaction, opacity tables, effects of diffusion, mixing etc. On the other hand, the parameter $f$ constitutes a new asteroseismic probe, giving information on subphotospheric layers and is complementary to oscillation frequencies determined by stellar interior. Comparing empirical and theoretical values of $f$, one can draw conclusions about the efficiency of convection in $\delta$ Sct stars (Daszyńska-Daszkiewicz et al 2003,2005b) or about opacities in $\beta$ Cep stars (Daszyńska-Daszkiewicz et al. 2005a).

\section{Uncertainties}

There are many sources of uncertainties in all results presented in this paper. Firstly, all calculation were done assuming the zero rotation approximation. Effects of rotation can spoil the nice mode separation in the diagnostic diagrams, making them dependent on the inclination, rotation rate and the azimuthal order $m$. One such effect is rotational mode coupling. It takes place if the frequency distance between modes, with the degrees $\ell$ differing by 2 and the same $m$, is of the order of the rotation frequency. In such a situation, the photometric amplitude for the coupled mode has to be calculated as superposition of all mode amplitudes satisfying the conditions $\ell_{k}=\ell_{j}+2$ and $m_{k}=m_{j}$. The effect of rotational mode coupling on the photometric diagrams for $\beta$ Cep star was studied by Daszyńska-Daszkiewicz et al. (2002). Examples for $\delta$ Sct stars can be found in Daszyńska-Daszkiewicz (2007). Another case when the rotation has to be included is when the pulsational frequency, $\omega$, is of the order of the rotational frequency, $\Omega$, so that the perturbation approach fails. It happens often in the case of the rapidly rotating SPB stars, in which high order gravity modes are excited. Such slow modes are no longer described by the spherical harmonics, and more complicated formalisms are needed. One possibility is the use of the traditional approximation which allows expressing the angular dependence of eigenfunctions by the Hough functions (e.g. Lee \& Saio 1997, Townsend 2003b). The formula for the light variation due to low frequency oscillation was given by Townsend (2003a). Daszyńska-Daszkiewicz et al. (2007) discussed a prospect for mode identification from diagnostic diagrams and derived the expression for the radial velocity variation.

Another uncertainty comes from the input physics and atmospheric models. The effect of metallicity parameter, $Z$, and opacities on the diagnostic diagrams for $\beta$ Cep star models was considered by Cugier et al. (1994). For a higher value of $Z$, the separation of modes with different $\ell$ 's is much better. Similarly, computations with the OP tables, instead of OPAL, lead to a little better $\ell$ dis- 
crimination, especially the radial modes are much more spread. Then, Cugier \& Daszyńska (2001) checked the effect of the atmospheric metallicity parameter, $[\mathrm{m} / \mathrm{H}]$, and the microturbulence velocity, $\xi_{t}$, on the diagnostic properties of photometric diagrams. For $\beta$ Cep stars these parameters have negligible impact.

The main problem in applying the method of diagnostic diagrams to $\delta$ Sct modes is that photometric amplitudes and phases exhibit a strong dependence on the treatment of convection (Balona \& Evers 1999). To circumvent this problem, Daszyńska-Daszkiewicz et al. (2003, 2005b) invented the method of simultaneous determination of degree $\ell$ and the nonadiabatic parameter $f$ from observations. Then we can identify the $\ell$-value independently of the pulsation models and, by comparing empirical and theoretical $f$ values, constraints on convection can be inferred. The result was that the convective transport in $\delta$ Sct stars studied by us is rather inefficient. The progress in modelling $\delta$ Sct pulsation with time-dependent convection treatment was achieved e.g. Grigahčene et al. (2005), Dupret et al. (2005a) and Dupret et al. (2005b).

In the case of $\delta$ Sct models, the uncertainties in atmospheric models can play much more important role than in the B-type pulsators. In Kurucz standard models, the flux derivatives over effective temperature and gravity are not smooth at the temperature where convective transport becomes important. This is because of using an overshooting approximation that moves the flux higher in the atmosphere, above the top of the nominal convection zone. In the NOVER-ODFNEW models computed by F. Castelli the problem of nonsmooth derivatives does not exist. There are also NEMO models (New Model Grid of Stellar Atmospheres) which include different treatment of convection. The models were computed by the Vienna group with modified versions of the Kurucz ATLAS9 code. The grids have smaller steps in $T_{\text {eff }}$ and $\log g$ than in Kurucz's computations, and the flux derivatives are perfectly smooth. The effect of using various atmospheric models in the calculation of $\delta$ Sct observables was discussed by Daszyńska-Daszkiewicz et al. (2004) and Daszyńska-Daszkiewicz (2007).

\section{Conclusions}

There is a potential for mode identification from the BRITE photometry. As we could see, two-colour information can yield some constraints on the spherical harmonic degree, $\ell$, of the oscillation modes excited in main sequence pulsators.

However, space observations should be followed up by simultaneous groundbased spectroscopy. The advantages of adding the spectroscopic variations is obvious. By combing photometry and the radial velocity data, we will improve significantly the discrimination of $\ell$ and infer better seismic constraints on stel- 
lar parameters and input physics. Moreover, from the line profile variations the identification of the azimuthal order, $m$, becomes possible. With such unprecedented data, we can hope for a great step in asteroseismology of main sequence pulsators with the BRITE-Constellation.

Acknowledgments. The author thanks Werner Weiss for inviting her to participate in the BRITE Workshop and Mikołaj Jerzykiewicz for carefully reading the manuscript. This work was supported by the Polish MNiSW grant No. 1 P03D 02128.

\section{References}

Balona, L.A., Evers, E.A. 1999, MNRAS 302, 349

Balona, L.A., Stobie, R.S. 1979, MNRAS 189, 649

Barban, C., Matthews, J.M., De Ridder, J. 2007, A\&A 468, 1033

Bruntt, H., Suárez, J.C., Bedding, T.R., et al. 2007, A\&A 461, 619

Castelli, F., Kurucz, R.L. 2004, in Proceedings of the IAU Symp. No 210, Modelling of Stellar Atmospheres, eds. N. Piskunov et al., poster A20

Cugier, H., Daszynska, J. 2001, A\&A 377, 113

Cugier, H., Dziembowski, W.A., Pamyantykh, A.A., 1994, A\&A 291, 143

Daszyńska-Daszkiewicz, J., Dziembowski, W.A., Pamyantykh, A.A., et al. 2002, A\&A 392, 151

Daszyńska-Daszkiewicz, J., Dziembowski, W.A., Pamyatnykh, A.A. 2003, A\&A 407, 999

Daszyńska-Daszkiewicz, J., Dziembowski, W.A., Pamyatnykh, A.A., et al. 2004, in Proceedings of the IAU Symp. No. 224, The A-Star Puzzle, eds. J. Zverko, J.

Ziznovsky, S.J. Adelman, and W.W. Weiss, p. 853

Daszyńska-Daszkiewicz, J., Dziembowski, W.A., Pamyatnykh, A.A., 2005a, A\&A 441,641

Daszyńska-Daszkiewicz, J., Dziembowski, W.A., Pamyatnykh, A.A., et al. 2005b, A\&A 438, 653

Daszyńska-Daszkiewicz, J., 2007, CoAst 150, 32

Daszyńska-Daszkiewicz, J., Dziembowski, W.A., Pamyatnykh, A.A. 2007, Acta Astron. 57, 11

Dziembowski, W.A. 1977, Acta Astron. 27, 203

Dupret, M.-A., Grigahcène, A., Garrido, R., et al. 2005a, A\&A 435, 927

Dupret, M.-A., Grigahcène, A., Garrido, R., et al. 2005b, MNRAS 361, 476

Garrido, R., Garcia-Lobo, E., Rodriguez, E. 1990, A\&A 234, 262

Grigahcène, A., Dupret, M.-A., Gabriel, M., et al. 2005, A\&A 434, 1055 
Heynderickx, D., Waelkens, C., Smeyers, P. 1994, A\&AS 105,447

Iglesias, C.A., Rogers, F.J. 1996, ApJ 464, 943

Nowakowski, R. 2005, Acta Astron. 55, 1

Saio, H., Cameron, C., Kuschnig, R., et al. 2007, CoAst 150, 215

Townsend, R.H.D. 2002, MNRAS 330, 855

Townsend, R.H.D. 2003a, MNRAS 343, 125

Townsend, R.H.D. 2003b, MNRAS, 340, 1020

Walker, G.A.H., Kuschnig, R., Matthews, J.M., et al. 2005, ApJ, 635, L77

Watson, R.D. 1988, Ap\&SS 140, 255 